\title{
Original Article \\ Carthamus tinctorius Enhances the Antitumor Activity of Dendritic Cell Vaccines via Polarization toward Th1 Cytokines and Increase of Cytotoxic T Lymphocytes
}

\author{
Jia-Ming Chang, Le-Mei Hung, Yau-Jan Chyan, Chun-Ming Cheng, and Rey-Yuh Wu \\ Division of Research and Development, Development Center for Biotechnology, Xizhi City, Taipei County, Taiwan 221, R.O.C., Taiwan
}

Correspondence should be addressed to Jia-Ming Chang, jiaming@ntu.edu.tw

Received 19 March 2008; Accepted 9 October 2008

Copyright ( $) 2011$ Jia-Ming Chang et al. This is an open access article distributed under the Creative Commons Attribution License, which permits unrestricted use, distribution, and reproduction in any medium, provided the original work is properly cited.

\begin{abstract}
Carthamus tinctorius (CT), also named safflower, is a traditional Chinese medicine widely used to improve blood circulation. CT also has been studied for its antitumor activity in certain cancers. To investigate the effects of CT on the dendritic cell (DC)-based vaccine in cancer treatment, cytokine secretion of mouse splenic T lymphocytes and the maturation of DCs in response to CT were analyzed. To assess the antitumor activity of CT extract on mouse CD117 ${ }^{+}$(c-kit)-derived DCs pulsed with JC mammal tumor antigens, the JC tumor was challenged by the CT-treated DC vaccine in vivo. CT stimulated IFN- $\gamma$ and IL- 10 secretion of splenic T lymphocytes and enhanced the maturation of DCs by enhancing immunological molecule expression. When DC vaccine was pulsed with tumor antigens along with CT extract, the levels of TNF- $\alpha$ and IL- $1 \beta$ were dramatically increased with a dosedependent response and more immunologic and co-stimulatory molecules were expressed on the DC surface. In addition, CTtreated tumor lysate-pulsed DC vaccine reduced the tumor weight in tumor-bearing mice by $15.3 \%$ more than tumor lysate-pulsed DC vaccine without CT treatment. CT polarized cytokine secretion toward the Th1 pathway and also increased the population of cytotoxic T lymphocytes ex vivo. In conclusion, CT activates DCs might promote the recognition of antigens and facilitate antigen presentation to Th1 immune responses.
\end{abstract}

\section{Introduction}

Carthamus tinctorius (CT), also named safflower, is a traditional Chinese medicine widely used to improve blood circulation [1], extending the coagulation time in mice and exhibiting a significant antithrombotic effect [2]. However, CT is used not only for its traditional medicinal purposes but is also effective for treating breast cancer [3]. The oil extracted from the seed of CT is reported to contain alkane-6,8-diols, which have the activity to inhibit $12-\mathrm{O}$ tetradecanoylphorbol-13-acetate-induced tumor promotion in two-stage carcinogenesis in mouse skin. In addition, $\mathrm{N}$ feruloylserotonin and $N$-( $p$-coumaroyl) serotonin strongly inhibit the melanin production of Streptomyces bikiniensis and B16 melanoma cells $[4,5]$. These compounds are suggested to have potential antitumor effects. In addition, CT also is neuroprotective for cerebral ischemic injury in vivo and in vitro [2]. Recently, $\mathrm{N}$-( $p$-coumaroyl)serotonin and $\mathrm{N}$-( $p$-coumaroyl)tryptamine, active ingredients in CT, were shown to strongly inhibit the production of proinflammatory cytokines (IL- $1 \alpha$, IL- $1 \beta$, IL-6, IL- 8 and TNF$\alpha$ ) from lipopolysaccharide-stimulated human monocytes [6]. Notably, extracts of CT exhibit a vast diversity of bioactivities, including immunomodulation, anti-infarction, antiallergic, anti-inflammatory and antiestrogenic effects, as well as functioning as a hemostatic agent to promote blood coagulation. Table 1 summarizes the diverse bioactivities of CT and delineates the therapeutic implications for this potent herbal medicine.

Dendritic cells (DCs) are professional antigen-presenting cells that stimulate immune responses by presenting endogenous or exogenous antigens to T-helper lymphocytes [31]. Immature DCs can be differentiated from monocytes and bone marrow progenitor cells by treatment with GM-CSF and IL-4 [32, 33], after which the cells gain strong phagocytic activity but not antigen presentation activity. Immature DCs are stimulated with maturation signals to express more surface immunological molecules (such as CD86, CD80, 
TABLE 1: The biological activities and therapeutic indications for extracts of CT.

\begin{tabular}{|c|c|c|}
\hline Plant part & $\begin{array}{l}\text { Possible active } \\
\text { ingredients }\end{array}$ & Biological activities and clinical relevance \\
\hline \multicolumn{3}{|l|}{ Flower } \\
\hline & \multirow[t]{2}{*}{ Safflor yellow } & $\begin{array}{l}\text { Inhibitory effects on the production of antibody and delayed-type } \\
\text { hypersensitivity reaction in vivo; and the responsiveness of mixed } \\
\text { lymphocyte reaction and production of IL } 2 \text { in vitro }\end{array}$ \\
\hline & & Antilipid peroxidation effects \\
\hline & Triterpene & $\begin{array}{l}\text { Anti-inflammatory activity against } \\
\text { 12-O-tetradecanoylphorbol-13-acetate-induced inflammation }\end{array}$ \\
\hline & Polysaccharides & $\begin{array}{l}\text { Antitumor activities through toll-like receptor } 4 / \mathrm{NF}-\kappa \mathrm{B} \text { pathway in } \\
\text { the cell assay }\end{array}$ \\
\hline & Hydroxysafflor yellow A & $\begin{array}{l}\text { Anti-thrombotic and -infraction effects and as a neuroprotective } \\
\text { agent against cerebral ischemic damage }\end{array}$ \\
\hline & \multirow[t]{3}{*}{$\begin{array}{l}\text { Carthamin, safflower } \\
\text { yellow }\end{array}$} & Antiproliferative and pro-apoptotic activities in hepatic stellate cells \\
\hline & & $\begin{array}{l}\text { Reduction of cerebral infarction volume along with increase of bcl-2 } \\
\text { and decrease of caspase- } 3\end{array}$ \\
\hline & & $\begin{array}{l}\text { Improving functions of cardiac contraction and dilation, increasing } \\
\text { coronary blood flow and strengthening the bcl-2 protein expression }\end{array}$ \\
\hline & \multirow[t]{2}{*}{$\begin{array}{l}\text { Water soluble } \\
\text { substances }\end{array}$} & Cytotoxic effects on the rat nervous cells and may cause teratogenesis \\
\hline & & Antimycotic properties especially against Aspergillus fumigatus \\
\hline
\end{tabular}

Seed

Oil extracts

Ethanol-ethyl acetate extract (serotonin derivatives and their glucoside)

Serotonin derivatives

Trechelosides

Linoleate

Leaf

Flavonoids

Kinobeon A
Antitumor effects in a skin and beast cancer animal model and in a melanoma cell assay system

Immunomodulatory effects mainly associated with anti-inflammatory activities

Antioxidant effects mainly for prevention of atherosclerosis through inhibition of LDL oxidation and postischemic myocardial dysfunction

Anabolic effects and antiestrogenic activities on bone through promotion of osteoblastic differentiation and inhibition of bone resorption

High linoleate in dietary oil supplements increase serum concentrations of prostaglandin F $2 \alpha$ metabolite

Antioxidative effects

A preventive effect on singlet oxygen and acting as a tyrosinase inhibitor
Literature source

Lu et al. [7]

Jin et al. [8]

Akihisa et al. [9]

Ando et al. [10]

Wang et al. [1]

Zhu et al. [2]

Zhang et al. [11]

Zhu et al. [12, 13]

Chor et al. [14]

Luo et al. [15]

Zhang and Jiang [16]

Nobakht et al. [17]

Blaszczyk et al. [18]

Loo et al. [3]

Roh et al. [4]

Yasukawa et al. [5]

Takii et al. [6]

Koyama et al. [19]

Hotta et al. [20]

Kim et al. [21]

Jang et al. [22]

Yoo et al. [23]

Yuk et al. [24]

Hong et al. [25]

Grant et al. [26]

Lee et al. [27]

Kambayashi et al. [28] Kanehira et al. [29, 30]
MHC-I and MHC-II) for antigen presentation, leading to a strong immune response against foreign antigens [34]. TNF- $\alpha$ and CD40L can stimulate immature DCs to mature in vitro and may show potential for clinical use [35], suggesting that enhancement of DC maturation is required for improvement of DC immunotherapy. In the clinic, DC-based immunotherapies are being intensively studied for their application in the treatment of certain diseases, including cancer and infectious diseases [36-38]. Scientists are trying to improve the phagocytic activity or antigen presentation activity of DCs for use in immunotherapy [39, 40]. Certain herbal medicines can also improve DC function [41]. To date, CT has been used as a folk medicine in cancer adjuvant therapy, but its function has not been proven. In this study, we studied the immunomodulatory effects of CT on cytokine secretion and surface immunologic molecules of DCs in vitro and antitumor activities of CT-treated DCs in an animal model of breast cancer. 


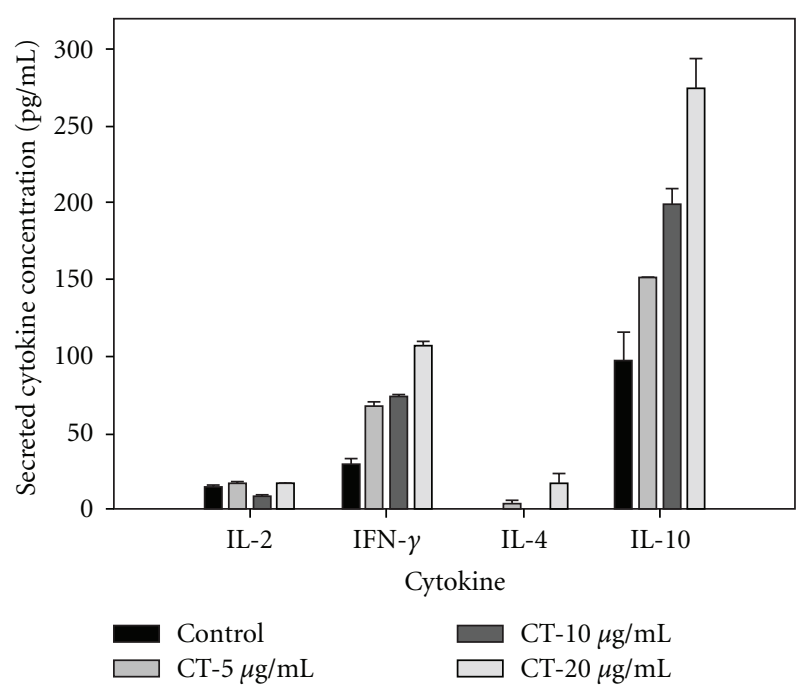

FIGURE 1: CT extract stimulates cytokine secretion of mouse splenic T lymphocytes. Splenocytes were homogenized from the spleen of healthy BALB/c mice. Mouse splenic T lymphocytes were treated with various concentrations of CT extract for $24 \mathrm{~h}$, and then the medium was assessed for secretion of IL-2, IFN- $\gamma$, IL-4 and IL-10, as determined by ELISA. The assay was performed in triplicate, and the data are expressed as mean \pm SE.

\section{Materials and Methods}

2.1. Cell Cultures and Reagents. JC cells (mouse mammary adenocarcinoma) were purchased form American Type Culture Collection (ATCC, USA) and cultured in MEM medium (Gibco, Invitrogen Inc., USA) supplemented with 10\% fetal bovine serum (FBS) (Hyclone, USA). GM-CSF and IL-4 were purchased from the PeproTech Inc. (NJ, USA). Phycoerythrin (PE)-hamster antimouse CD80 (B7-1) (16-10A1 clone, Cat No. 553769), fluorescein isothiocyanate (FITC)rat antimouse CD86 (B7-2) (GL1 clone, Cat. No. 553691), PE-mouse antimouse $\mathrm{H}-2 \mathrm{~K}^{\mathrm{d}}$ (MHC-I) (SF1-1.1 clone, Cat. No. 553566) and FITC-mouse antimouse I-A ${ }^{\mathrm{d}}$ (MHC-II) (AMS-32.1 clone, Cat No. 553547) were purchased from Becton Dickinson-Pharmingen (NJ, USA). FITC-anti-CD3 and PE-anti-CD8 were purchased from Becton DickinsonPharmingen. To prepare the CT plant extract used in our experiments, the flowers of the plants were collected and dried. Dried flowers $(100 \mathrm{~g})$ were boiled in 11 distilled water $(1: 10$ ratio, w/v) for $1 \mathrm{~h}$ and the extract was filtered through Whatman No. 1 filter paper (Whatman, USA). The filtrate was lyophilized into powder and stored at $4^{\circ} \mathrm{C}$. The CT powder was dissolved in phosphate-buffered saline (PBS) ( $\mathrm{pH} 7.4$ ) at a stock concentration of $100 \mathrm{mg} / \mathrm{mL}$ for in vitro experiments.

2.2. Cytokine Secretion from Mouse Splenocytes Induced by CT Extract. The splenocytes were homogenized from the spleen of healthy BALB/c mice, and the splenic T lymphocytes were isolated by passage through nylon wool columns [42]. The purity of $\mathrm{T}$ cells was confirmed by flow cytometry to be $>90 \%$. The mouse splenic T lymphocytes were treated with various concentrations of CT extract $(5,10$ and $20 \mu \mathrm{g} / \mathrm{mL}$ of culture medium) for $24 \mathrm{~h}$. The medium was collected to determine the concentrations of the cytokines IL-2, IFN- $\gamma$ and IL-4. Cytokine levels were determined by the DuoSet ELISA kit as described in the manual.

2.3. Maturation of Mouse DCs by CT Extract. Bone marrow cells were taken from BALB/c mice and then suspended in RPMI-1640 medium containing 10\% FBS. The cell suspension was allowed to stand at room temperature for $10 \mathrm{~min}$ to remove the cell clots, and then the cell suspension was centrifuged at $800 \mathrm{~g}$ for $5 \mathrm{~min}$ at $4^{\circ} \mathrm{C}$ and washed with PBS (pH 7.4) containing $0.5 \%$ FBS and $2 \mathrm{mM}$ EDTA. The cells were allowed to attach to a $100-\mathrm{mm}$ culture plate for $2 \mathrm{~h}$ and the floating cells were removed gently. The cells were then treated with GM-CSF $(100 \mathrm{ng} / \mathrm{mL})$ and IL-4 $(100 \mathrm{ng} / \mathrm{mL})$ at $37^{\circ} \mathrm{C}, 5 \% \mathrm{CO}_{2}$ for 6 days, following the modifications of Zheng et al. [43]. After 6 days of differentiation, the DCs were treated with various concentrations of CT extract $(5,10$ and $20 \mu \mathrm{g} / \mathrm{mL}$ of culture medium) for $72 \mathrm{~h}$. After CT treatment, the DCs were collected for flow cytometric analysis of surface immunological molecules (CD80, CD86, MHC-I and MHCII). In brief, the cells were stained with the specific FITCconjugated antibody.

2.4. Isolation of $\mathrm{CD}_{117^{+}}$Bone Marrow Cells. Bone marrow cells were collected as above and suspended in PBS ( $\mathrm{pH} 7.4)$ containing $0.5 \% \mathrm{FBS}$ and $2 \mathrm{mM}$ EDTA prior to the isolation of $\mathrm{CD} 117^{+}$cells. $\mathrm{CD} 117^{+}$cells were isolated using magneticactivated cell sorting (Miltenyi Biotec Inc, CA, USA) as described in the manual. Briefly, $1 \times 10^{7}$ cells were added to $20 \mu \mathrm{l}$ anti-CD $117^{+}$provided by the kit for $15 \mathrm{~min}$ and then washed with PBS to remove excess unbound antibodies. The treated cells were loaded on a column, from which the CD117 ${ }^{-}$cells were eluted with PBS buffer in the presence of a magnetic field. The CD117 ${ }^{+}$cells were then eluted in the absence of a magnetic field and were then differentiated to DCs by adding GM-CSF and IL-4 as described above.

2.5. Generation of $C D 117^{+}$-Derived DCs. The CD $117^{+}$cells were cultured at a density of $6.25 \times 10^{7} /$ well of a 6 well plate in RPMI-1640 medium containing 10\% FBS, $1.5 \mathrm{mg} / \mathrm{mL}$ sodium bicarbonate, $0.1 \mu \mathrm{mol} / \mathrm{mL}$ nonessential amino acids, $1 \mu \mathrm{mol} / \mathrm{mL}$ sodium pyruvate, $100 \mathrm{U} / \mathrm{mL}$ penicillin $\mathrm{G}$ and $100 \mu \mathrm{g} / \mathrm{mL}$ streptomycin supplemented with GM-CSF $(100 \mathrm{ng} / \mathrm{mL})$ and IL-4 $(100 \mathrm{ng} / \mathrm{mL})$ at $37^{\circ} \mathrm{C}, 5 \%$ $\mathrm{CO}_{2}$ for 6 days. Medium containing GM-CSF and IL- 4 was refreshed every 2 days.

2.6. Assessment of Cytokines Released from JC Lysate-Pulsed $C T$-Treated $C D 117^{+}$-Derived DCs. After differentiation of CD $117^{+}$cells to DCs for 6 days, DCs were pulsed with JClysate and treated with or without CT extract (5, 10 and $20 \mu \mathrm{g} / \mathrm{mL}$ ) for an additional 3 days. The conditioned medium was collected for determination of the concentrations of IL2 , IL-10, TNF- $\alpha$ and IL- $1 \beta[44]$.

2.7. Assessment of Immunological Molecules of JC LysatePulsed CT-Treated CD $117^{+}$-Derived DCs. After differentiation of CD $117^{+}$cells to DCs for 6 days, DCs were pulsed with 

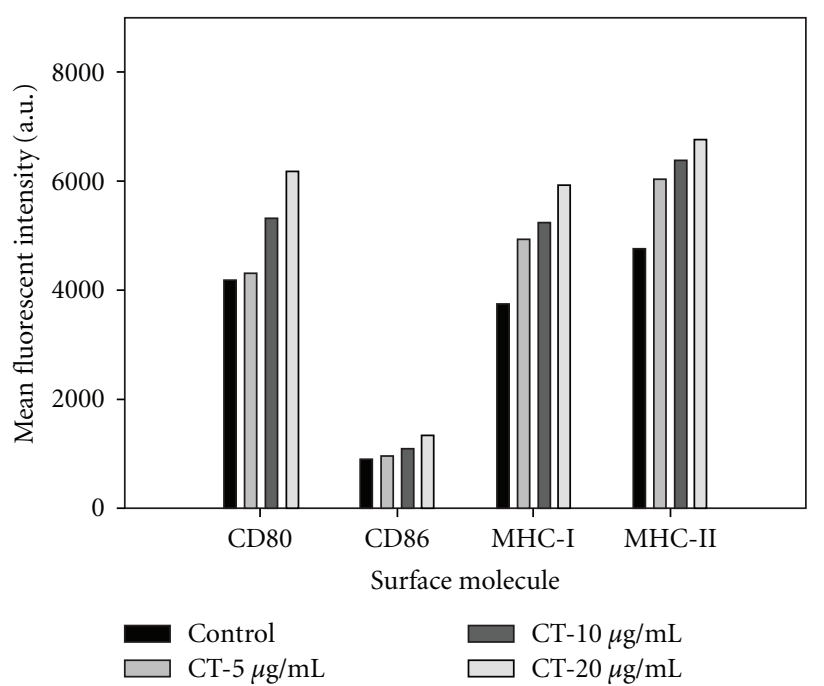

FIgURE 2: CT extract enhances the maturation of mouse DCs. Mouse bone marrow cells were collected as described in Section 2. After 6 days of differentiation with GM-CSF and IL-4, the cells were treated with various concentrations of CT extract in the absence of GM-CSF and IL-4 for an additional $48 \mathrm{~h}$. The cells were then scraped and collected for surface molecule analysis (CD80, CD86, MHC-I and MHC-II) using flow cytometry.

JC lysate and treated with or without CT extract $(20 \mu \mathrm{g} / \mathrm{mL})$ for an additional $72 \mathrm{~h}$. After CT treatment, DCs cell were analyzed for cell surface markers (CD80, CD86, MHC-I and anti-MHC-II) by flow cytometry.

2.8. Preparation of DC Vaccine and JC Tumor Animal Experiment. CD $117^{+}$cells were cultured in differentiation medium (GM-CSF and IL-4) for 6 days followed by cultivation in fresh RPMI medium in the presence or absence of CT $(20 \mu \mathrm{g} / \mathrm{mL})$ for 3 days. JC cells $\left(3-4 \times 10^{6}\right)$ were lyzed in $1 \mathrm{~mL}$ RPMI-1640 medium containing 10\% FBS by freezing and thawing four times, and the lysate was stored at $-80^{\circ} \mathrm{C}$ as a tumor antigen resource [45] to pulse the DCs.

$\mathrm{BALB} / \mathrm{c}$ female mice (4-6 weeks) were purchased from the National Laboratory Animal Center (Taiwan, ROC). JC cells $\left(3 \times 10^{6}\right.$ per $150 \mu \mathrm{l}$ PBS $)$ were subcutaneously inoculated in the flank of BALB/c mice. Twenty-four tumorbearing mice were grouped ( $n=8$ /group) as follows: untreated control, DC vaccine and CT-treated groups. In the DC vaccine group and CT-treated DC vaccine group, mice were injected intraperitoneally with the JC-pulsed DC cell suspension $\left(1.7 \times 10^{6}\right.$ per $\left.0.2 \mathrm{~mL} \mathrm{PBS}\right)$ and the CT-treated JC-pulsed DC cell suspension $\left(1.7 \times 10^{6}\right.$ per $0.2 \mathrm{~mL}$ PBS $)$ on day 13 , respectively. The tumor growth of JC tumor-bearing mice was measured by a caliper every $4-6$ days and calculated using the equation, tumor weight $(\mathrm{mg})=$ length $(\mathrm{mm}) \times$ width $\left(\mathrm{mm}^{2}\right) / 2$, until the tumor reached $2 \times 10^{3} \mathrm{~mm}^{3}$. On day 32, mice were euthanized by $\mathrm{CO}_{2}$, the tumors were excised and weighed and the spleens of each mouse were homogenized into a single cell suspension pulsed with or without CT-treated DCs to measure Th1- and Th2-related cytokines. The splenocytes were also re-stimulated with the previous corresponding JC-pulsed DC vaccine and CTtreated JC-pulsed DC vaccine for flow cytometric analysis of cytotoxic T lymphocytes. All mice received humane care, and the study protocol followed the guidelines of the Institutional Animal Care and Use Committees of the Development Center for Biotechnology (accredited by AAALAC).

2.9. Effects of CT-Treated DCs on the Cytokine Secretion and Cytotoxic T Lymphocytes of Mouse Splenocytes. DC vaccine was prepared as described above and cultured in AIM-V medium (Gibco, USA), and splenic T lymphocytes were taken from the normal and the JC tumor-bearing mice and cultured in RPMI-1640 medium (Gibco, USA). In brief, splenic T lymphocytes were partially purified using a nylon wool column [46]. Different cytokines in response to CT $(20 \mu \mathrm{g} / \mathrm{mL})$-treated DCs were determined when DCs $(2 \times$ $10^{5} / \mathrm{mL}$ ) were pulsed with splenocytes from tumor-bearing mice at a cell: cell ratio of $1: 10,1: 20$ and $1: 40$ for $48 \mathrm{~h}$ at $37^{\circ} \mathrm{C}, 5 \% \mathrm{CO}_{2}$. After cocultivation of splenocytes and DCs, the medium was collected and stored at $-20^{\circ} \mathrm{C}$ prior to cytokine analysis. Cytokines were analyzed by ELISA as described above. The splenocyte culture was continued for an additional $72 \mathrm{~h}$ and then subjected to flow cytometric analysis, in which specific $\mathrm{T}$ lymphocytes were gated by staining with FITC-anti-CD3 and PE-anti-CD8.

2.10. Statistical Analysis. Data were analyzed by one-way ANOVA using SPSS statistical software followed by the Dunnett's test for multiple comparisons between each group. A $P$-value $<.05$ was considered to indicate a significant difference.

\section{Results}

3.1. IFN- $\gamma$ and $I L-10$ Secretion from Mouse Splenic $T$ Lymphocytes. To determine the effect of CT extract on the stimulation of mouse resting $\mathrm{T}$ lymphocytes, the splenic $\mathrm{T}$ lymphocytes were partially purified and treated with various concentrations of CT extract for $24 \mathrm{~h}$. The supernatants were collected and evaluated for the levels of IL-2, IFN- $\gamma$ and IL4 and IL-10. Figure 1 shows that CT extract stimulated the production of IL-10 > IFN- $\gamma$ in a dose-dependent manner (Figure 1). However, no such stimulation was observed for IL-2 and IL-4.

3.2. Expression of Immunological Molecules in the Immature DCs. To determine whether the CT extract stimulated the maturation of DCs, mouse bone marrow cells were isolated and differentiated into immature DCs by adding GM-CSF and IL-4 for 6 days. The immature DCs were treated with various concentrations of CT extract $(5,10$ and $20 \mu \mathrm{g} / \mathrm{mL})$ for $48 \mathrm{~h}$. Figure 2 shows that CT extract promoted the expression of DC surface markers (CD86 < MHC-I = MHC$\mathrm{II}=\mathrm{CD} 80)$ in a dose-dependent manner (Figure 2).

3.3. Enhancement of Maturation of DCs by CT Extract. To study whether the pattern of cytokine secretion was altered in DCs stimulated by CT extract in the presence of tumor 


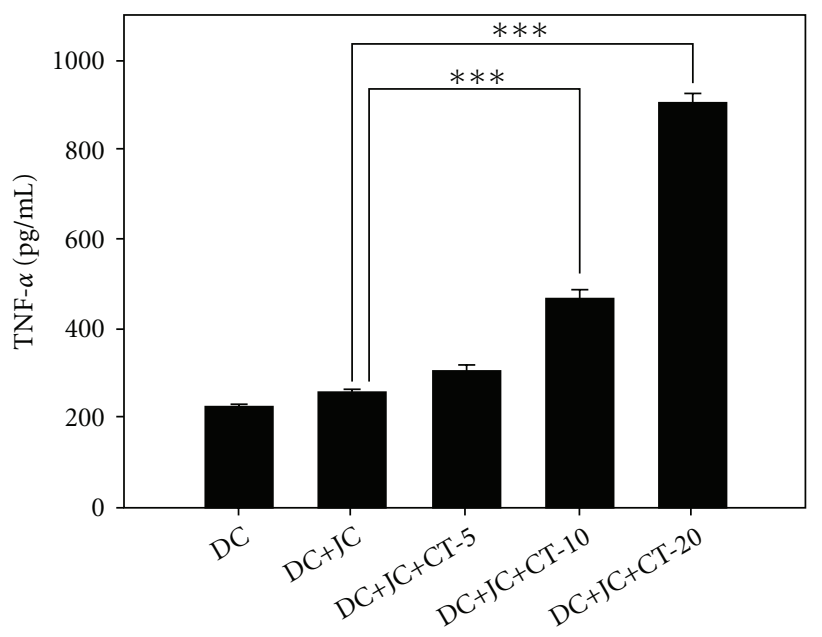

(a)

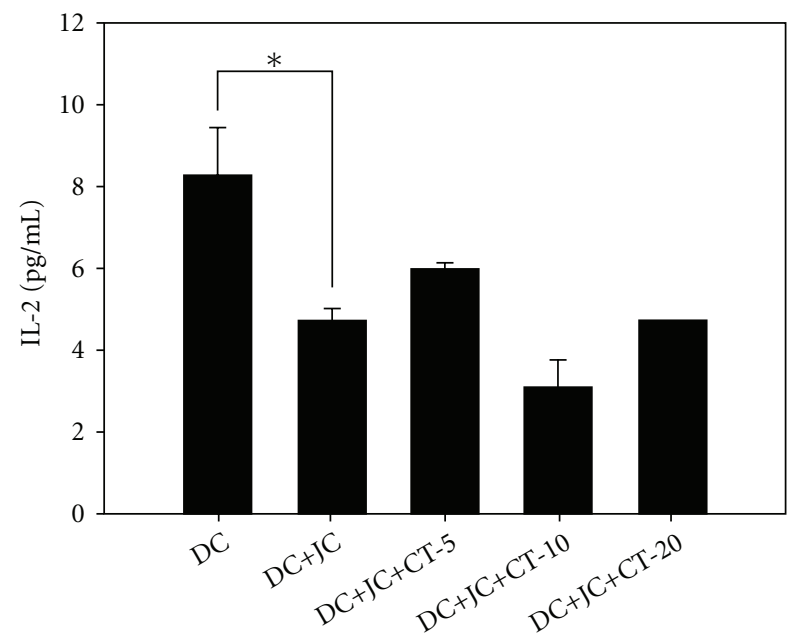

(c)

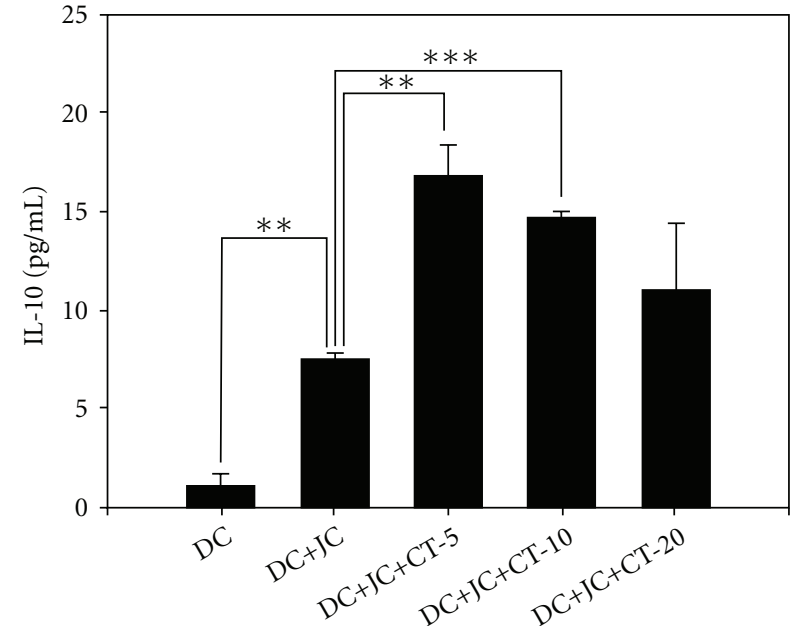

(b)

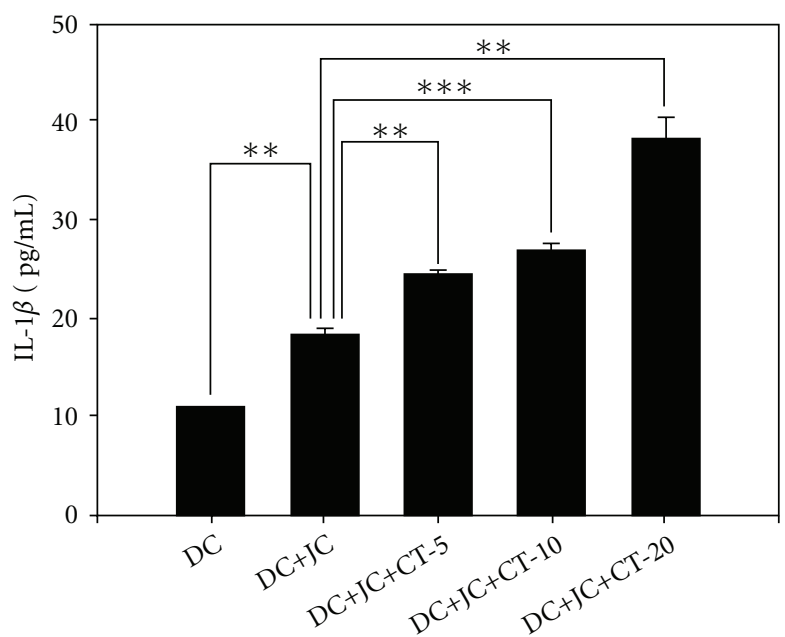

(d)

FIGURE 3: CT extract enhances the maturation of $\mathrm{CD} 117^{+}$-derived DCs in the presence of tumor antigen. Mouse bone marrow cells were collected as described in Section 2. After 6 days of differentiation with GM-CSF and IL-4, the cells were pulsed with JC tumor lysate $(1.7 \times$ $10^{6}$ per $0.2 \mathrm{~mL}$ PBS; DC + JC) and treated with various concentrations of CT extract $(5,10$, and $20 \mu \mathrm{g} / \mathrm{mL} ; \mathrm{DC}+\mathrm{JC}+\mathrm{CT}-5, \mathrm{DC}+\mathrm{JC}+$ CT-10 and DC + JC + CT-20) in the absence of GM-CSF and IL-4 for additional 48 h. The medium was collected, and the concentrations of secreted cytokines were determined. Data from triplicate points are expressed as the mean \pm SE. Statistical analysis was performed by oneway ANOVA followed by Dunnett's test. A $P$-value $<.05$ was considered to reflect a significant difference. ${ }^{*} P<.05,{ }^{* *} P<.01,{ }^{* * *} P<.001$.

antigens, the immature DCs were pulsed with JC tumor lysate along with different concentrations of CT extract (5, 10 and $20 \mu \mathrm{g} / \mathrm{mL}$ ) for $48 \mathrm{~h}$. The conditioned medium was analyzed for IL-2, IL-10, TNF- $\alpha$ and IL- $1 \beta$ by ELISA and the DCs were subjected to analysis of surface immunological molecules (CD80, CD86, MHC-I and MHC-II). The results showed that CT extract increased TNF- $\alpha$ and IL- $1 \beta$ levels in a dose-dependent manner (Figure 3). However, the level of IL-2 was reduced when DCs were pulsed with tumor lysate. IL-10 decreased with an increase in the concentration of CT extract, suggesting that CT extract might increase the secretion of TNF- $\alpha$ and IL- $1 \beta$ to enhance the maturation of immature DCs. Treatment with CT extract maintained the high profile of cell surface markers, such as CD80, CD86,
MHC-I and MHC-II on the CD117+-derived CDs in the presence of tumor antigen (Figure 4).

\subsection{Inhibition of Tumor Growth by CT Treated-DC Vaccine.} To study the antitumor activity of CT-treated DC vaccine, $\mathrm{BALB} / \mathrm{c}$ mice were inoculated with JC tumor cells and challenged with a single dose of CT-treated DC vaccine. The JC tumor-bearing mice were challenged with DC vaccine and CT-treated DC vaccine on day 13, when tumor reached the size of $5 \mathrm{~mm}$ (length) $\times 5 \mathrm{~mm}$ (width). Results showed that CT-treated DC vaccine and DC vaccine decreased the tumor mass by $32.5 \%$ and $17.2 \%$, respectively, compared to the untreated control (Figure 5). This result suggested that CT might increase the antitumor activity of the DC vaccine. 


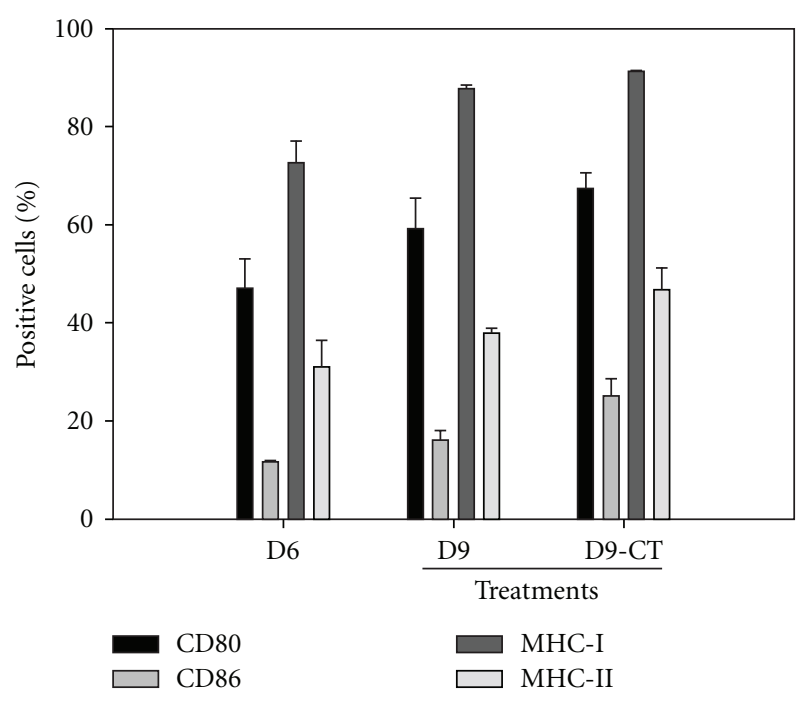

FIGURE 4: CT extract enhances the maturation of CD117 $7^{+}$-derived DCs. CD $117^{+}$cells were isolated as described in Section 2 and then cultured in differentiation medium (GM-CSF and IL-4). After 6 days of differentiation, the differentiated cells (D6) were pulsed with JC tumor lysate $\left(1.7 \times 10^{6}\right.$ per $0.2 \mathrm{~mL}$ PBS $)$ in the absence of differentiation cytokines for additional $72 \mathrm{~h}$ (D9) or pulsed with JC tumor lysate and incubated with $20 \mu \mathrm{g} / \mathrm{mL}$ CT for additional $72 \mathrm{~h}$ (D9-CT). The CD117 ${ }^{+}$-derived DCs were then collected and analyzed by flow cytometry for the expression of surface immunological molecules. Experiments were performed in triplicate, and the data are expressed as mean \pm SE.

3.5. Polarization of Cytokine Secretion to Th1 Response. To determine the Th1 and Th2 cytokine secretion in DC vaccine treatment, the tumor-bearing mice that had been challenged with DC vaccine were sacrificed and their splenocytes subjected to cytokine analysis, in which those cells were restimulated with the previous corresponding DC vaccine or CT-treated DC vaccine ex vivo. Splenocytes of the CT/DC vaccine compared with those of the CT-untreated DCvaccine showed increased or identical production of IL-2, IL-10 and IFN- $\gamma$. However, IL-4 production was notably decreased in splenocytes of mice treated with the CT/DC vaccine (Figure 6). The secretion of cytokines was not increased in the sham splenocytes when those cells pulsed with DC vaccine or CT-treated DC vaccine with lack of JC antigen. This result suggested that either the DC vaccine or CT-treated DC vaccine elicited antitumor activity through a Th1 response, and that CT extract might also enhance the immune response of the DC vaccine.

3.6. Increased Antitumor Activity Is Mediated by Cytotoxic T Lymphocytes. To determine whether cytotoxic T lymphocytes were increased in the CT-treated DC vaccine, the splenocytes of vaccine-challenged mice were re-stimulated with the previous corresponding DC vaccine or CT-treated DC vaccine. The JC tumor lysate-pulsed DC vaccine stimulated $30.1 \%$ of cytotoxic T lymphocytes and the JC tumor lysate-pulsed CT-treated DC vaccine stimulated $35.6 \%$ of

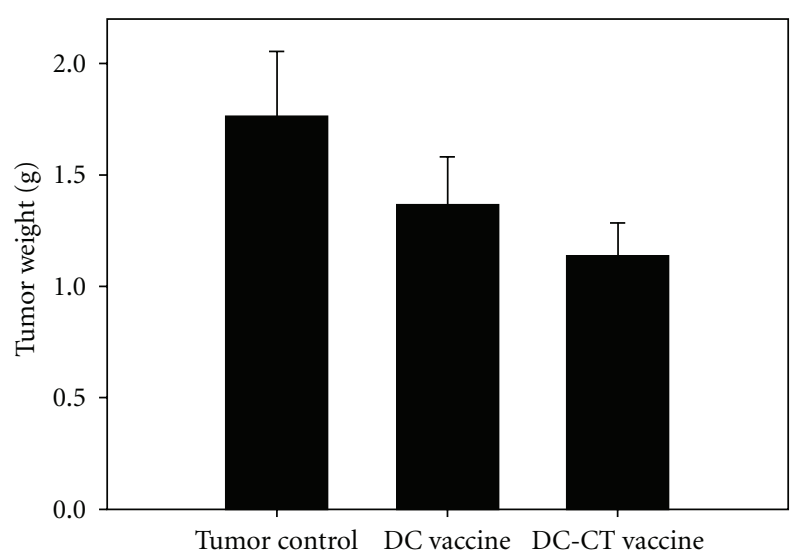

FIGURE 5: Antitumor activity of the CT extract-treated DC vaccine in JC tumor-bearing mice. DC vaccine or CT-treated DC vaccine (DC-CT) was administered via intraperitoneal injection on day 13 when mean estimated tumor weight was $>63 \mathrm{mg}(0.5 \times 0.5 \mathrm{~cm}$ in diameter). A group of tumor-bearing animals without treatment was the tumor control. Animals were sacrificed on day 32, and the tumors were excised and weighed. Data are expressed as mean $\pm \mathrm{SE}$ ( $n=8$ in each group).

cytotoxic $\mathrm{T}$ lymphocytes (Figure 7), indicating that CTtreated JC tumor lysate-pulsed DC vaccine could increase more cytotoxic $\mathrm{T}$ lymphocytes against tumors than $\mathrm{DC}$ vaccine without CT treatment. Additionally, the cytotoxic T lymphocytes of sham mice were only stimulated $27.6 \%$ with CT-treated DC vaccine in the absence of JC tumor antigens. This result was consistent with the decrease in tumor weight shown in Figure 5.

\section{Discussion}

DCs are professional antigen-presenting cells that have been enlisted for use in vaccines against cancer. DCs can acquire tumor antigens by cocultivation with tumor cells or pulsation with tumor lysates to become activated. After maturation, the DCs present the tumor antigens to lymphocytes and trigger the immune response cascade. In this study, CT stimulated splenic T lymphocytes to secrete IFN- $\gamma$ and IL-10, suggesting that CT can immunomodulate $\mathrm{T}$ lymphocyte function. Heightened expressions of CD80, CD86, MHC-I and MHC-II imply that CT stimulates maturation of antigen-presenting cells; moreover, an increase in the expression of MHC-I and II molecules, together with an increase in CD8-positive T cells, suggests that HLA-mediated presentation of tumor antigens accelerates after treatment with CT extract. CT also improved the antitumor activity of the DC vaccine as evidenced by a reduction in tumor weight. The ex vivo analysis of cytokine secretion and lymphocyte population suggested that CT polarizes the immune response toward the Th1 pathway by increasing the secretions of IL-2 and IFN- $\gamma$, but not IL-4, and consequently produces more cytotoxic $\mathrm{T}$ lymphocytes to elicit antitumor activity than DC vaccine without CT treatment. It has been reported that stimulated DCs secrete TNF- $\alpha$, IL- $1 \beta$ and IL-10 during the 


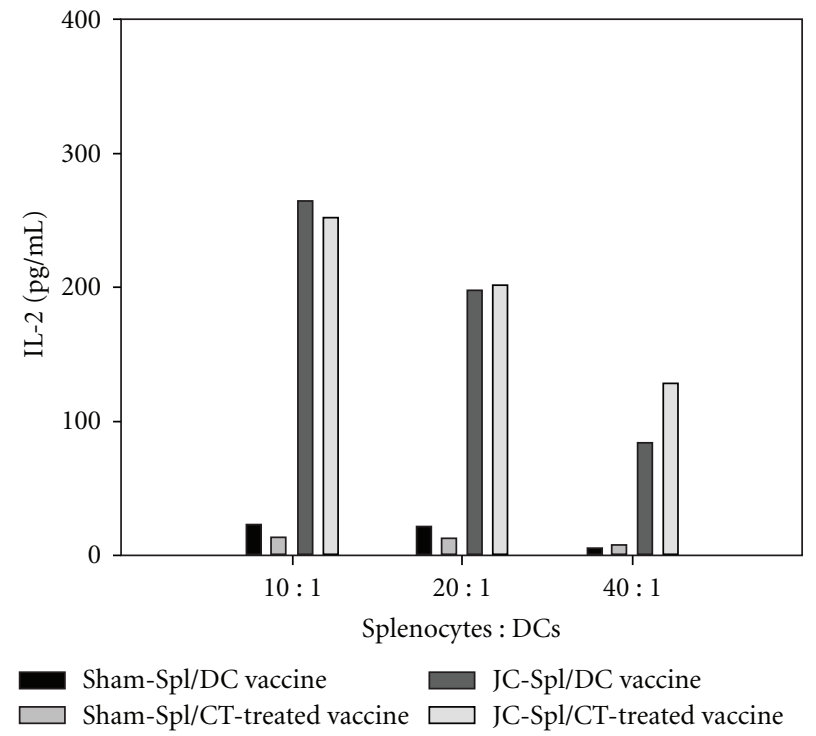

(a)

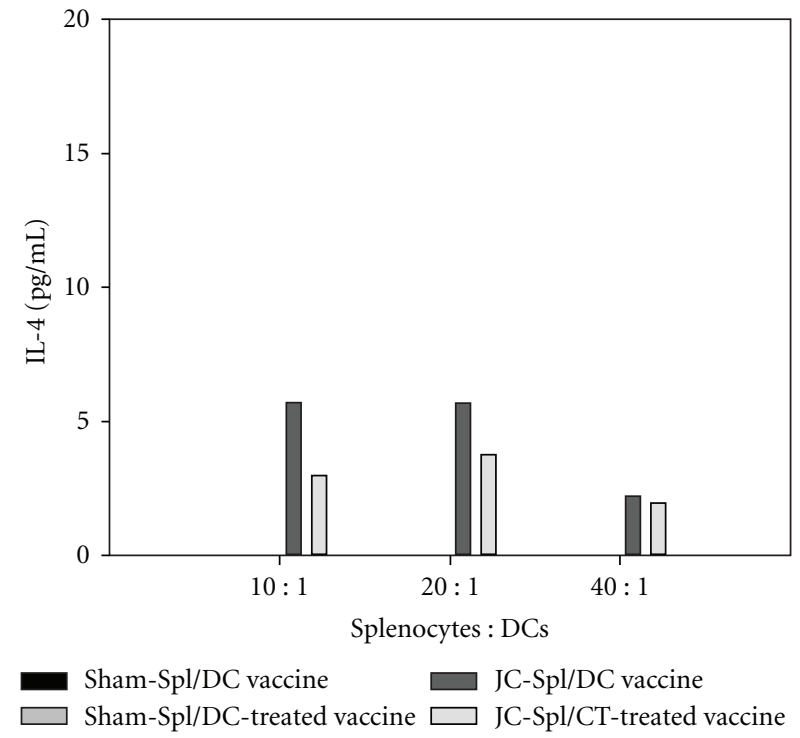

(c)

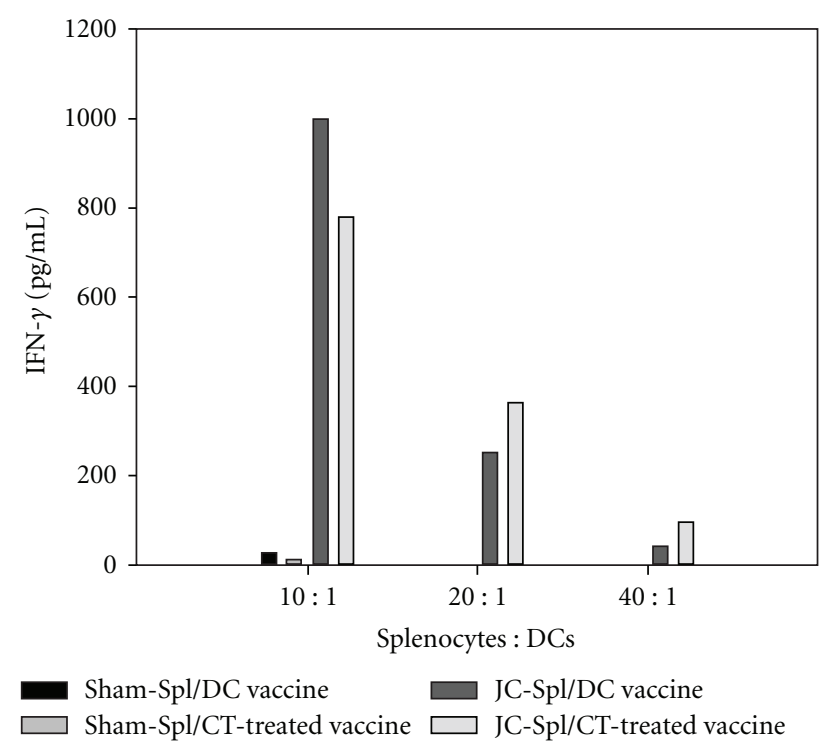

(b)

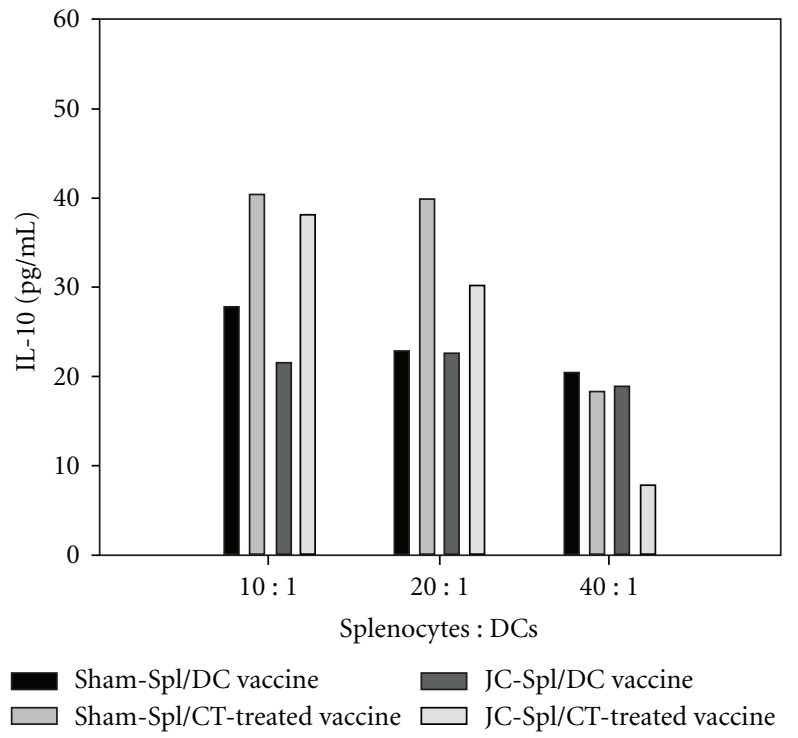

(d)

FIGURE 6: Secretion of cytokines from mouse splenocytes re-stimulated with CT extract-treated DC vaccine ex vivo. Spleens from sham (sham-spl) and tumor-bearing (JC-spl) BALB/c mice challenged with DC vaccine or CT-treated DC vaccine were taken and homogenized into single cell suspensions. The splenocytes were cocultured with DC vaccine or CT-treated DC at cell : cell ratios of 10:1, 20:1 and 40:1 for $48 \mathrm{~h}$. The concentrations of secreted cytokines were determined by ELISA as described in Section 2.

maturation process and then polarize the $\mathrm{T}$ lymphocytes toward the Th1 pathway [47], which is consistent with our observations shown in Figure 3.

DCs are widely distributed in the human body with different morphologies, such as Kupffer cells in liver [48] and Langerhans cells in the skin [49]. Unlike chemotherapy which produces severe side effects during treatment, the use of DCs provides an alternative strategy against tumors [50]. The functions of DCs are improved in such as the maturation, antigen presentation and regulatory cytokine secretion, which has survival benefits in cancer patients [51]. Although the CT-treated DC vaccine was intraperitoneally injected to activate immunity in this study, oral administration of CT extract might promote the recognition of antigens and facilitate antigen presentation via intestinal DCs, and thus this attractive approach is worthy of further investigation. However, many traditional medicinal herbals have being studied for immunomodulatory activities, such as Melilotus suaveolens Ledeb [52] and Tanacetum parthenium [53]. In literatures, both herbal plants were found to demonstrate their anti-inflammation activities in a monocytic cellbased assay system and exhibited effects on regulating the production of chemokines. Due to these recent studies, $M$. suaveolens Ledeb [52] and T. parthenium have been 


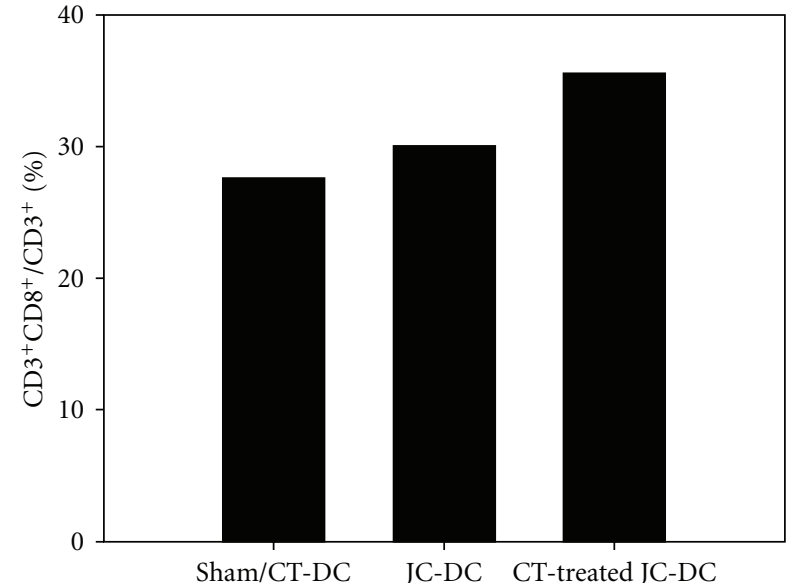

Figure 7: Ex vivo stimulation of specific T lymphocytes. The spleens of sham and tumor-bearing mice were taken and homogenized into single-cell suspensions. The splenocytes were re-stimulated with their corresponding treatments (DC vaccine or CT-treated DC vaccine) for 5 days. The splenocytes were then subjected to flow cytometric analysis, and specific T lymphocytes were gated by staining with FITC-anti-CD3 and PE-anti-CD8.

implicated to exhibit potential therapeutic benefits in treating many inflammation-related diseases, such as cancers, atherosclerosis and rheumatoid arthritis. Therefore, herbals in treating cancers might have activities via the modulation of cytokine profiles. In this article, our results suggest that CT could promote immunity through the activation of DCs per se that do not alter the cytokine secretion during immune responses of tumor lysate-pulsed DCs toward the Th1 pathway.

\section{References}

[1] Z. T. Wang, S. R. Wang, and M. J. Zhao, "Comparative study on effect of recipe for activating blood circulation and replenishing Qi on left ventricular remodeling in rats with left heart failure after myocardial infarction," Zhongguo Zhong Xi Yi Jie He Za Zhi, vol. 22, no. 5, pp. 376-378, 2002.

[2] H.-B. Zhu, L. Zhang, Z.-H. Wang et al., “Therapeutic effects of hydroxysafflor yellow A on focal cerebral ischemic injury in rats and its primary mechanisms," Journal of Asian Natural Products Research, vol. 7, no. 4, pp. 607-613, 2005.

[3] W. T. Y. Loo, M. N. B. Cheung, and L. W. C. Chow, "The inhibitory effect of a herbal formula comprising ginseng and carthamus tinctorius on breast cancer," Life Sciences, vol. 76, no. 2, pp. 191-200, 2004.

[4] J. S. Roh, J. Y. Han, J. H. Kim, and J. K. Hwang, "Inhibitory effects of active compounds isolated from safflower (Carthamus tinctorius L.) seeds for melanogenesis," Biological and Pharmaceutical Bulletin, vol. 27, no. 12, pp. 1976-1978, 2004.

[5] K. Yasukawa, T. Akihisa, Y. Kasahara et al., "Inhibitory effect of alkane-6,8-diols, the components of safflower, on tumor promotion by 12-O-tetradecanoylphorbol-13-acetate in twostage carcinogenesis in mouse skin," Oncology, vol. 53, no. 2, pp. 133-136, 1996.
[6] T. Takii, S. Kawashima, T. Chiba et al., "Multiple mechanisms involved in the inhibition of proinflammatory cytokine production from human monocytes by $\mathrm{N}$-(pcoumaroyl)serotonin and its derivatives," International Immunopharmacology, vol. 3, no. 2, pp. 273-277, 2003.

[7] Z.-W. Lu, F. Liu, J. Hu, D. Bian, and F.-G. Li, "Suppressive effects of safflower yellow on immune functions," Acta Pharmacologica Sinica, vol. 12, no. 6, pp. 537-542, 1991.

[8] M. Jin, J. R. Li, and W. Wu, "Study on the antioxidative effect of Safflor Yellow," Zhongguo Zhong Yao Za Zhi, vol. 29, no. 5, pp. 447-449, 2004.

[9] T. Akihisa, K. Yasukawa, H. Oinuma et al., "Triterpene alcohols from the flowers of compositae and their antiinflammatory effects," Phytochemistry, vol. 43, no. 6, pp. 12551260, 1996.

[10] I. Ando, Y. Tsukumo, T. Wakabayashi et al., "Safflower polysaccharides activate the transcription factor NF- $\kappa \mathrm{B}$ via Toll-like receptor 4 and induce cytokine production by macrophages," International Immunopharmacology, vol. 2, no. 8, pp. 11551162, 2002.

[11] J. C. Zhang, L. Xia, and M. Bai, "Changes of ICAM-1 and P-selectin in rats with pulmonary thromboembolism and the effect of safflower injection," Zhongguo Zhong Xi Yi Jie He Za Zhi, vol. 26, no. 7, pp. 629-632, 2006.

[12] H. Zhu, Z. Wang, C. Ma et al., "Neuroprotective effects of hydroxysafflor yellow a: in vivo and in vitro studies," Planta Medica, vol. 69, no. 5, pp. 429-433, 2003.

[13] H.-B. Zhu, Z.-H. Wang, J.-W. Tian, F.-H. Fu, K. Liu, and C.-L. Li, "Protective effect of hydroxysafflor yellow A on experimental cerebral ischemia in rats," Yao Xue Xue Bao, vol. 40, no. 12, pp. 1144-1146, 2005.

[14] S. Y. Chor, A. Y. Hui, K. F. To et al., "Anti-proliferative and proapoptotic effects of herbal medicine on hepatic stellate cell," Journal of Ethnopharmacology, vol. 100, no. 1-2, pp. 180-186, 2005.

[15] J. Luo, Z. P. Fang, L. M. Zhou, and S. T. Lai, "Effects of Carthamus tinctorius injection on bcl-2, caspase- 3 expression related to neurons apoptosis after local cerebral ischemia," Zhongguo Zhong Yao Za Zhi, vol. 29, no. 10, pp. 977-980, 2004.

[16] S. Q. Zhang and L. D. Jiang, "Effect of safflower injection on cardiac energy charge and anti-apoptosis gene bcl-2 in rats' heart," Zhongguo Zhong Xi Yi Jie He Za Zhi, vol. 24, no. 5, pp. 442-444, 2004.

[17] M. Nobakht, M. Fattahi, M. Hoormand, I. Milanian, N. Rahbar, and M. Mahmoudian, "A study on the teratogenic and cytotoxic effects of safflower extract," Journal of Ethnopharmacology, vol. 73, no. 3, pp. 453-459, 2000.

[18] T. Blaszczyk, J. Krzyzanowska, and E. Lamer-Zarawska, "Screening for antimycotic properties of 56 traditional Chinese drugs," Phytotherapy Research, vol. 14, no. 3, pp. 210-212, 2000.

[19] N. Koyama, K. Kuribayashi, T. Seki et al., "Serotonin derivatives, major safflower (Carthamus tinctorius L.) seed antioxidants, inhibit Low-Density Lipoprotein (LDL) oxidation and atherosclerosis in apolipoprotein E-deficient mice," Journal of Agricultural and Food Chemistry, vol. 54, no. 14, pp. 49704976, 2006.

[20] Y. Hotta, A. Nagatsu, W. Liu et al., "Protective effects of antioxidative serotonin derivatives isolated from safflower against postischemic myocardial dysfunction," Molecular and Cellular Biochemistry, vol. 238, no. 1-2, pp. 151-162, 2002.

[21] K.-W. Kim, S.-J. Suh, T.-K. Lee et al., "Effect of safflower seeds supplementation on stimulation of the proliferation, 
differentiation and mineralization of osteoblastic MC3T3-E1 cells," Journal of Ethnopharmacology, vol. 115, no. 1, pp. 4249, 2008.

[22] H. O. Jang, Y. S. Park, J. H. Lee et al., "Effect of extracts from safflower seeds on osteoblast differentiation and intracellular calcium ion concentration in MC3T3-E1 cells," Natural product research, vol. 21, no. 9, pp. 787-797, 2007.

[23] H. H. Yoo, J. H. Park, and S. W. Kwon, "An anti-estrogenic lignan glycoside, tracheloside, from seeds of Carthamus tinctorius," Bioscience, Biotechnology and Biochemistry, vol. 70, no. 11, pp. 2783-2785, 2006.

[24] T. H. Yuk, J. H. Kang, S. R. Lee et al., "Inhibitory effect of Carthamus tinctorius L. seed extracts on bone resorption mediated by tyrosine kinase, COX-2 (cyclooxygenase) and PG (prostaglandin) E2," American Journal of Chinese Medicine, vol. 30, no. 1, pp. 95-108, 2002.

[25] H.-T. Hong, H.-J. Kim, T.-K. Lee et al., "Inhibitory effect of a Korean traditional medicine, Honghwain-Jahage (water extracts of Carthamus tinctorius L. seed and Hominis placenta) on interleukin-1-mediated bone resorption," Journal of Ethnopharmacology, vol. 79, no. 2, pp. 143-148, 2002.

[26] M. H. J. Grant, B. M. Alexander, B. W. Hess et al., "Dietary supplementation with safflower seeds differing in fatty acid composition differentially influences serum concentrations of prostaglandin F metabolite in postpartum beef cows," Reproduction Nutrition Development, vol. 45, no. 6, pp. 721727, 2005.

[27] J. Y. Lee, E. J. Chang, H. J. Kim, J. H. Park, and S. W. Choi, "Antioxidative flavonoids from leaves of Carthamus tinctorius," Archives of Pharmacal Research, vol. 25, no. 3, pp. 313-319, 2002.

[28] Y. Kambayashi, S. Takekoshi, M. Nakano, M. Shibamori, Y. Hitomi, and K. Ogino, "Kinobeon A, purified from cultured safflower cells, is a novel and potent singlet oxygen quencher," Acta Biochimica Polonica, vol. 52, no. 4, pp. 903-907, 2005.

[29] T. Kanehira, S. Takekoshi, H. Nagata et al., "A novel and potent biological antioxidant, Kinobeon A, from cell culture of safflower," Life Sciences, vol. 74, no. 1, pp. 87-97, 2003.

[30] T. Kanehira, S. Takekoshi, H. Nagata, R. Y. Osamura, and T. Homma, "Kinobeon A as a potent tyrosinase inhibitor from cell culture of safflower: in vitro comparisons of kinobeon A with other putative inhibitors," Planta Medica, vol. 69, no. 5, pp. 457-459, 2003.

[31] E. Gilboa, "DC-based cancer vaccines," Journal of Clinical Investigation, vol. 117, no. 5, pp. 1195-1203, 2007.

[32] R. Paillot, F. Laval, J.-C. Audonnet, C. Andreoni, and V. Juillard, "Functional and phenotypic characterization of distinct porcine dendritic cells derived from peripheral blood monocytes," Immunology, vol. 102, no. 4, pp. 396-404, 2001.

[33] T. Schroeder, C. Lange, J. Strehl, and U. Just, "Generation of functionally mature dendritic cells from the multipotential stem cell line FDCP-mix," British Journal of Haematology, vol. 111, no. 3, pp. 890-897, 2000.

[34] R. M. Steinman, "Dendritic cells and the control of immunity: enhancing the efficiency of antigen presentation," Mount Sinai Journal of Medicine, vol. 68, no. 3, pp. 160-166, 2001.

[35] G. M. Zou and Y. K. Tam, "Cytokines in the generation and maturation of dendritic cells: recent advances," European Cytokine Network, vol. 13, no. 2, pp. 186-199, 2002.

[36] P. J. Mosca, H. K. Lyerly, T. M. Clay, M. A. Morse, and H. K. Lyerly, "Dendritic cell vaccines," Frontiers in Bioscience, vol. 12, pp. 4050-4060, 2007.
[37] N. Meidenbauer, R. Andreesen, and A. Mackensen, "Dendritic cells for specific cancer immunotherapy," Biological Chemistry, vol. 382, no. 4, pp. 507-520, 2001.

[38] T. G. Berger and E. S. Schultz, "Dendritic cell-based immunotherapy," Current Topics in Microbiology and Immunology, vol. 276, pp. 163-197, 2003.

[39] D. Avigan, "Dendritic cells: development, function and potential use for cancer immunotherapy," Blood Reviews, vol. 13, no. 1, pp. 51-64, 1999.

[40] G. Hartmann, G. J. Weiner, and A. M. Krieg, "CpG DNA: a potent signal for growth, activation, and maturation of human dendritic cells," Proceedings of the National Academy of Sciences of the United States of America, vol. 96, no. 16, pp. 9305-9310, 1999.

[41] Y. L. He, Y. Ying, Y. L. Xu, J. F. Su, H. Luo, and H. F. Wang, "Effects of Lycium barbarum polysaccharide on tumor microenvironment T-lymphocyte subsets and dendritic cells in H22-bearing mice," Zhong Xi Yi Jie He Xue Bao, vol. 3, no. 5, pp. 374-377, 2005.

[42] L. G. Radvanyi, G. B. Mills, and R. G. Miller, "Religation of the $\mathrm{T}$ cell receptor after primary activation of mature $\mathrm{T}$ cells inhibits proliferation and induces apoptotic cell death," Journal of Immunology, vol. 150, no. 12, pp. 5704-5715, 1993.

[43] Z. Zheng, M. Takahashi, M. Narita et al., "Generation of dendritic cells from adherent cells of cord blood by culture with granulocyte-macrophage colony-stimulating factor, interleukin-4, and tumor necrosis factor- $\alpha$," Journal of Hematotherapy and Stem Cell Research, vol. 9, no. 4, pp. 453-464, 2000.

[44] J.-M. Chang, C.-M. Cheng, L.-M. Hung, Y.-S. Chung, and R.Y. Wu, "Potential use of Plectranthus amboinicus in the treatment of rheumatoid arthritis," Evidence-Based Complementary and Alternative Medicine, vol. 7, no. 1, pp. 115-120, 2010.

[45] U. S. Gaipl, L. E. Munoz, G. Grossmayer et al., "Clearance deficiency and systemic lupus erythematosus (SLE)," Journal of Autoimmunity, vol. 28, no. 2-3, pp. 114-121, 2007.

[46] D. Trizio and G. Cudkowicz, "Separation of T and B lymphocytes by nylon wool columns: evaulation of efficacy by functional assays in vivo," Journal of Immunology, vol. 113, no. 4, pp. 1093-1097, 1974.

[47] Y.-S. Wang, K.-H. Chi, and R.-M. Chu, "Cytokine profiles of canine monocyte-derived dendritic cells as a function of lipopolysaccharide- or tumor necrosis factor-alpha-induced maturation," Veterinary Immunology and Immunopathology, vol. 118, no. 3-4, pp. 186-198, 2007.

[48] T. C. R. Prickett, J. L. McKenzie, and D. N. J. Hart, "Characterization of interstitial dendritic cells in human liver," Transplantation, vol. 46, no. 5, pp. 754-761, 1988.

[49] T. Cao, H. Ueno, C. Glaser, J. W. Fay, A. K. Palucka, and J. Banchereau, "Both langerhans cells and intestinal DC crosspresent melanoma antigens and efficiently activate antigenspecific CTL," European Journal of Immunology, vol. 37, no. 9, pp. 2657-2667, 2007.

[50] P. A. MacAry, C. T. Too, and X. Dai, "Targeting tumours by adoptive transfer of immune cells," Clinical and Experimental Pharmacology and Physiology, vol. 33, no. 5-6, pp. 569-574, 2006.

[51] R. Hájek and A. W. Butch, "Dendritic cell biology and the application of dendritic cells to immunotherapy of multiple myeloma," Medical Oncology, vol. 17, no. 1, pp. 2-15, 2000.

[52] L. Zhao, J.-Y. Tao, S.-L. Zhang, F. Jin, R. Pang, and J.-H. Dong, "N-butanol extract from melilotus suaveolens Ledeb affects pro- and anti-inflammatory cytokines and mediators," 
Evidence-Based Complementary and Alternative Medicine, vol. 7, no. 1, pp. 97-106, 2010.

[53] C.-F. Chen and C.-H. Cheng, "Regulation of cellular metabolism and cytokines by the medicinal herb feverfew in the human monocytic THP-1 Cells," Evidence-Based Complementary and Alternative Medicine, vol. 6, no. 1, pp. 91-98, 2009. 


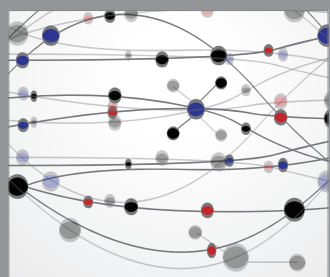

The Scientific World Journal
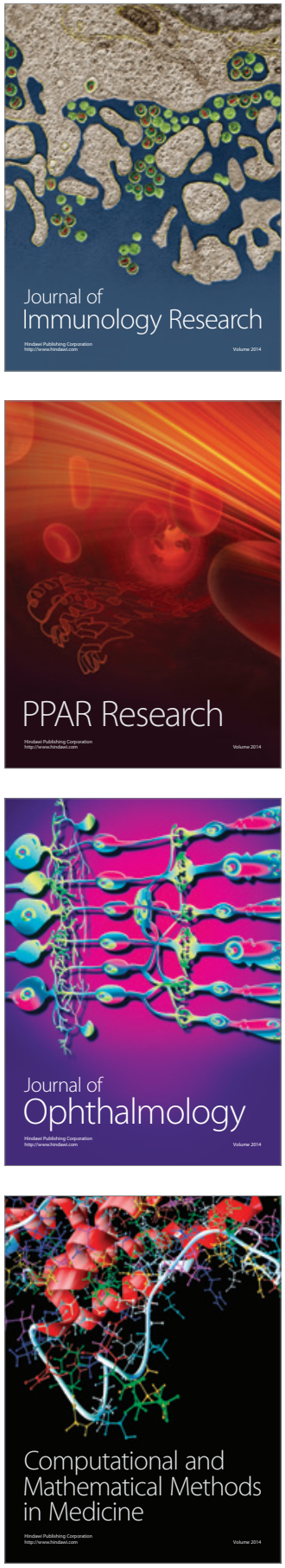

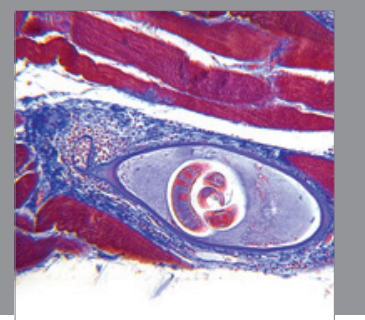

Gastroenterology

Research and Practice
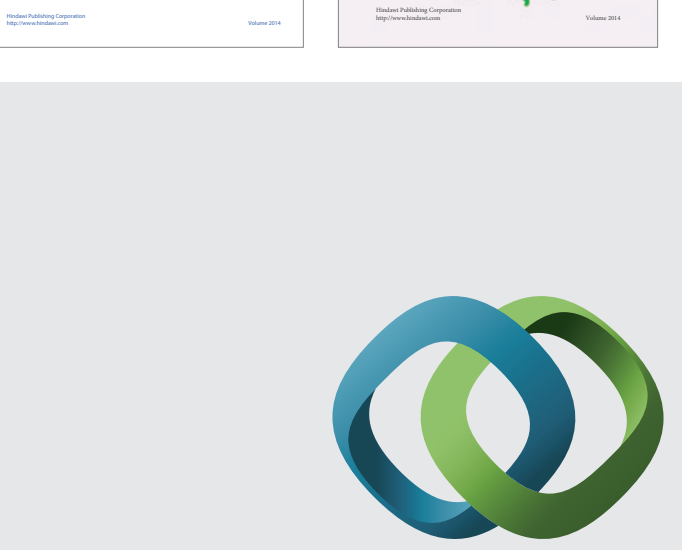

\section{Hindawi}

Submit your manuscripts at

http://www.hindawi.com
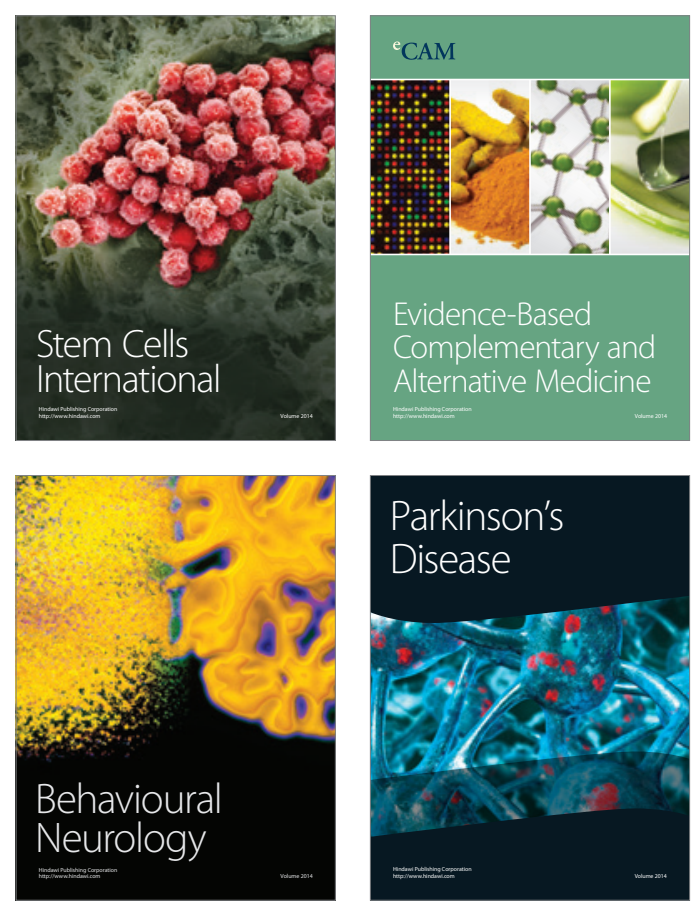

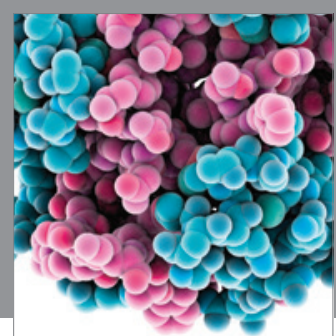

Journal of
Diabetes Research

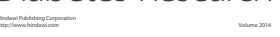

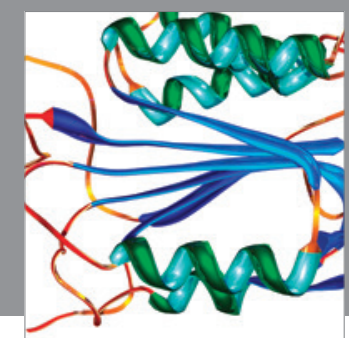

Disease Markers
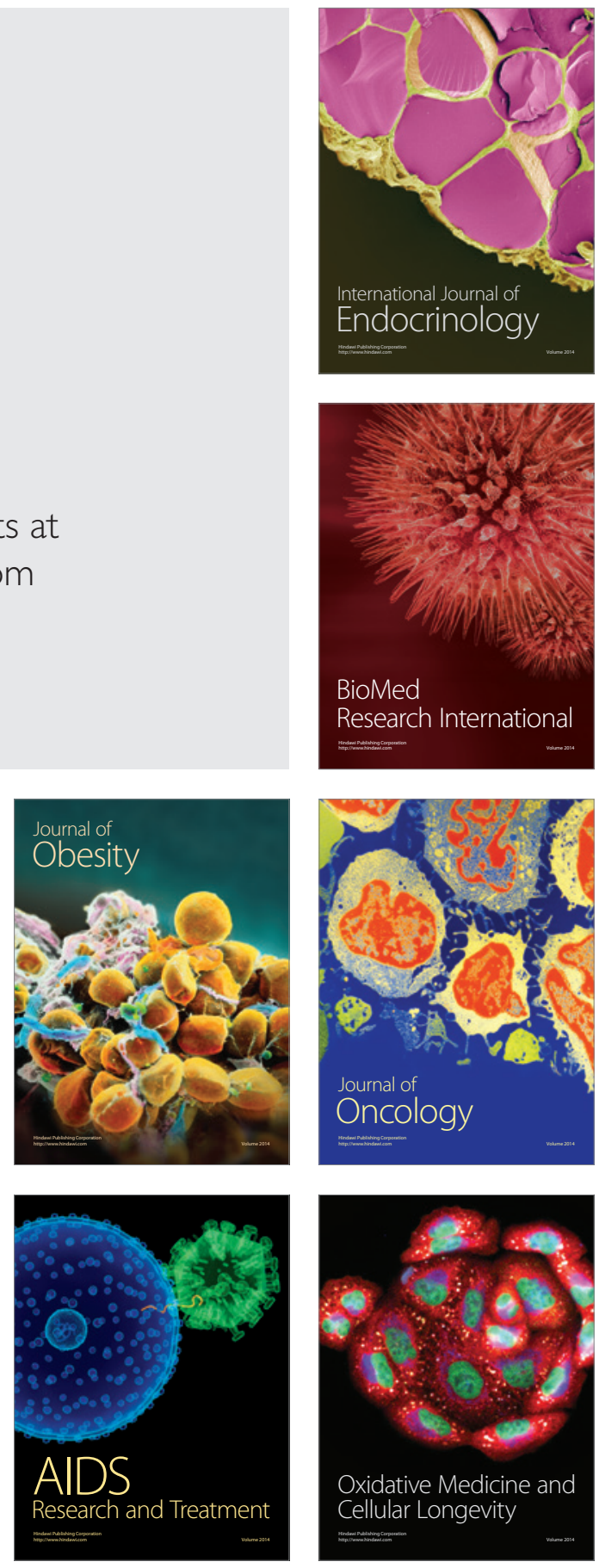